\title{
Octaarginine-modified gold nanoparticles enhance the radiosensitivity of human colorectal cancer cell line LSI80 to megavoltage radiation
}

This article was published in the following Dove Press journal: International Journal of Nanomedicine

\author{
Xuyang Zhang' \\ Hao Wang' \\ Jonathan Andrew Coulter ${ }^{2}$ \\ Ruijie Yang' \\ 'Department of Radiation Oncology, \\ Peking University Third Hospital, \\ Beijing, China; ${ }^{2}$ School of Pharmacy, \\ Queen's University of Belfast, \\ Belfast, UK
}

Background: This study investigated the effectiveness and underpinning mechanisms of radiosensitization using octaarginine (R8)-modified gold nanoparticle-poly(ethylene glycol) (GNP-PEG-R8) in colorectal cancer cell line LS180 to megavoltage radiotherapy in vitro.

Method: In-house synthesized GNP-PEG was characterized by transmission electron microscopy, dynamic light scattering, ultraviolet-visible spectrophotometry, and X-ray photoelectron spectroscopy. Inductively coupled plasma mass spectroscopy was used to quantify internalization. Direct cytotoxicity was established using the Cell Counting Kit-8, while radiosensitivity was determined using the gold standard in vitro clonogenic assay. Cell-cycle distribution, apoptosis, reactive oxygen species (ROS), and mitochondrial membrane potential (MMP) were analyzed by flow cytometry, further exploring the key mechanisms driving GNP-PEG-R8 radiosensitization.

Results: The core GNP diameter was $6.3 \pm 1.1 \mathrm{~nm}($ mean $\pm \mathrm{SD})$. Following functionalization, the hydrodynamic diameter increased to $19.7 \pm 2.8 \mathrm{~nm}$ and $27.8 \pm 1.8 \mathrm{~nm}$ for GNP-PEG and GNPPEG-R8, with respective surface plasmon resonance peaks of $515 \mathrm{~nm}$ and $525 \mathrm{~nm}$. Furthermore, incorporation of the R8 significantly increased nanoparticle internalization compared to GNPPEG $(p<0.001)$ over a $1 \mathrm{~h}$ treatment period. Functionalized GNPs confer little cytotoxicity below $400 \mathrm{nM}$. In clonogenic assays, radiation combined with GNP-PEG-R8 induced a significant reduction in colony formation compared with radiation alone, generating a sensitizer enhancement ratio of 1.59 . Furthermore, GNP-PEG-R8 plus radiation predominantly induced cell-cycle arrest in the G2/M phase, increasing G2/M stalling by an additional 10\% over GNPPEG, markedly promoting apoptosis $(p<0.001)$. Finally, ROS levels and alterations in MMP were investigated, indicating a highly significant $(p<0.001)$ change in both parameters following the combined treatment of GNP-PEG-R8 and radiation over radiation alone.

Conclusion: R8-modified GNPs were efficiently internalized by LS180 cells, exhibiting minimal cytotoxicity. This yielded significant radiosensitization in response to megavoltage radiation. GNP-PEG-R8 may enhance radiosensitivity by arresting cell cycle and inducing apoptosis, with elevated ROS identified as the likely initiator.

Keywords: gold nanoparticles, octaarginine, colorectal cancer, megavoltage radiotherapy, mechanisms, radiosensitization

\section{Introduction}

Nanotechnology is a rapidly growing field with applications relevant to the diagnosis and treatment of cancer. ${ }^{1-3}$ Among nanomaterials, gold nanoparticles (GNPs) have emerged as an attractive candidate material for use in combination with radiotherapy, owing to a range of unique physical and chemical properties. ${ }^{4-6}$ 
Globally, colorectal cancer is currently the third most common human cancer and the fourth most lethal with respect to cancer-related mortality. ${ }^{7}$ According to the National Comprehensive Cancer Network, radiotherapy plays an important role in the treatment of colorectal cancer. ${ }^{8}$ In recent years, technological advances have significantly improved the precision of radiotherapy delivery; however, a large proportion of colorectal cancer patients will still eventually suffer local recurrence with progression to systemic disease. ${ }^{9}$ In this regard, the development of safe and effective radiosensitizers is highly desirable. The mass energy absorption coefficient of gold is 100-150 times greater than that of soft tissue in the kiloelectronvolt energy range. Indeed, GNPs have been shown to enhance the sensitivity of HCT116 cells to $26 \mathrm{keV}$ $\mathrm{X}$-rays with a sensitizer enhancement ratio (SER) of 1.69 in vitro. ${ }^{10}$ However, megavoltage X-rays are widely used instead of kiloelectronvolt sources in clinical practice. It has been reported that GNPs exhibit efficacy using megavoltage radiation sources in breast, prostate, and lung cancer. ${ }^{11-13}$ However, whether GNPs hold potential in the megavoltage energy range for colorectal cancer remains unclear.

Despite the potential of GNPs as novel radiosensitizers, high treatment concentrations and long preincubation periods are required to achieve sufficient tumor cell internalization. To address this, further functionalization with novel ligands is required to increase cellular uptake efficiency, without compromising the favorable biocompatibility of gold. Cellpenetrating peptides (CPPs) are short peptides consisting of seven to 30 amino acids that can translocate cell membranes, transferring large biologically active molecules. ${ }^{14-16}$ Octaarginine peptide (R8; sequence RRRRRRRRCAL) is an arginine-dependent cationic CPP, previously shown to enhance drug delivery. ${ }^{17}$ As such, we modified the surface of the core GNP with R8 to improve uptake efficiency. In addition to R8, poly(ethylene glycol) (PEG) was also conjugated to improve stability and prevent particle agglomeration. In this study, we selected the colorectal cancer cell line LS180 as our model system, investigating the radiosensitizing potential and underlying mechanisms of R8-modified GNPs in response to megavoltage radiotherapy in vitro.

\section{Methods and materials Synthesis of GNP-PEG-R8}

Core sphere-shaped GNPs were synthesized by the classical chemical reduction method..$^{18}$ First, $4 \mathrm{~mL}$ of $1 \%$ trisodium citrate and $1 \mathrm{~mL} 1 \%$ tannic acid were added to $15 \mathrm{~mL}$ doubledistilled water, and the mixture was heated to $60^{\circ} \mathrm{C}$. This was then added to $80 \mathrm{~mL}$ of aqueous solution containing $1 \mathrm{~mL} 1 \% \mathrm{HAuCl}_{4}$, which was heated to boiling point.
After vigorous stirring for $30 \mathrm{~min}, 3 \mathrm{~mL}$ of aqueous thiolterminated PEG ( $1 \mathrm{mg} / \mathrm{mL}$, MW 2000; Shanghai ZZBio. Co., Shanghai, China) was added to the mixture at room temperature to create GNP-PEG. The resulting colloid solution was gently stirred for $24 \mathrm{~h}$ and concentrated using an ultrafiltration tube. Finally, $1 \mathrm{~mL}$ of R8 peptide $(1 \mathrm{mg} / \mathrm{mL}$; ChinaPeptide Co., Shanghai, China) was added to the solution and stirred for a further $24 \mathrm{~h}$, creating GNP-PEG-R8. All nanoparticle variants were subsequently stored at $4^{\circ} \mathrm{C}$, diluted in deionized water, where no aggregation was observed.

\section{Characterization of GNP-PEG-R8}

The ultraviolet-visible (UV-vis) absorbance spectra of GNPPEG and GNP-PEG-R8 were measured using UV-vis spectrophotometry within the wavelength region of $400-800 \mathrm{~nm}$. Surface charge and the hydrodynamic diameter of the nanoparticle variants were measured using a Malvern Zetasizer Nano-ZS 90 (Malvern Instruments, Malvern, UK), diluted in deionized water. Nanoparticle morphology was analyzed using transmission electron microscopy (TEM) (Hitachi HT7700; Hitachi, Tokyo, Japan). X-ray photoelectron spectroscopy (XPS) (ESCALAB 250XI; ThermoFisher Scientific, Waltham, MA, USA) analysis was used to verify whether PEG and R8 were conjugated on to the GNP surface.

\section{Cell culture}

The human colorectal cancer cell line LS180 was kindly gifted by the Institute of Zoology, Chinese Academy of Sciences. In brief, LS180 cells were maintained in minimum Eagle's medium (MEM) supplemented with $100 \mathrm{IU} / \mathrm{mL}$ penicillin, $100 \mathrm{mg} / \mathrm{mL}$ streptomycin, $10 \%$ fetal bovine serum, $1 \%$ glutamine, and $1 \%$ sodium pyruvate in a humidified atmosphere containing $5 \% \mathrm{CO}_{2}$ at $37^{\circ} \mathrm{C}$.

\section{Cytotoxicity assessment}

LS180 cells were seeded in 96-well plates at a density of $5 \times 10^{3}$ per well and incubated overnight. Culture medium was then replaced with $100 \mu \mathrm{L}$ of fresh medium containing varying concentrations $(100-1,600 \mathrm{nM})$ of GNP-PEG or GNP-PEG-R8 for $12 \mathrm{~h}$. The Cell Counting Kit-8 (Dojindo Molecular Technologies, Kumamoto, Japan) assay was used to determine the cell viability, according to the manufacturer's instructions.

\section{Cellular uptake}

Cells $\left(1 \times 10^{6} \mathrm{LS} 180\right)$ suspended in $3 \mathrm{~mL}$ complete medium were seeded into $6 \mathrm{~cm}$ Petri dishes until $70 \%-80 \%$ confluence was achieved. GNP-PEG and GNP-PEG-R8 were added to the growth medium, achieving a final concentration of $400 \mathrm{nM}$. After incubation for various durations $(0.25,0.5$, 
$1,2,4,6$, and $12 \mathrm{~h}$ ), cells were collected and resuspended in PBS to a final volume of $5 \mathrm{~mL}$. Cells were then counted and dissolved in aqua regia. Internal gold concentration was subsequently quantified using inductively coupled plasma mass spectrometry (ICP-MS). Calculation of GNP number per cell was determined by converting gold atoms per sample to number of GNPs using the following mathematical derivation:

$$
\begin{gathered}
U=\frac{2}{3} \pi\left(\frac{D}{\mathrm{a}}\right)^{3} \\
N=\frac{M}{U}
\end{gathered}
$$

where $U$ represents the number of atoms in each GNP and $D$ is the diameter of the GNPs. The constant "a" refers to the edge of the gold unit cell, which has a value of $4.076 \AA$, with four gold atoms per unit cell. $N$ is the number of GNPs for the analyzed sample and $M$ is the number of gold atoms determined from ICP-MS measurement.

\section{Clonogenic assay}

First, $1 \times 10^{6}$ LS180 cells were seeded into $6 \mathrm{~cm}$ Petri dishes and allowed to attach overnight. GNP-PEG or GNP-PEG-R8 was added to cells at a final concentration of $400 \mathrm{nM}$ for $1 \mathrm{~h}$. Excess non-internalized nanoparticles were removed from the medium, cells were washed in PBS, and fresh medium was added. As soon afterwards as practically possible, cells were irradiated with $6 \mathrm{MV}$ X-rays, delivering total doses of $0,2,4$, 6,8 , and $10 \mathrm{~Gy}$, in a single fraction. One day post-irradiation, cells were transferred at low seeding densities to six-well plates to allow single-cell colony formation over a 14 day period. Cells were subsequently washed, fixed with methanol, and stained using $1 \%$ methylene blue. Colonies were manually scored, defining a colony as containing a minimum of 50 cells. Plating efficiency, survival fraction $(S F)$, and other radiobiological parameters, including average lethal dose $\left(D_{0}\right)$, quasi-threshold dose $\left(D_{\mathrm{q}}\right)$, and $\mathrm{SER}_{D 0 / D q / \mathrm{SF}}$, were calculated. The dose-survival curve was fitted using a single-hit multi-target statistical model. Data from three independent experimental replicates were analyzed for each group.

\section{Flow cytometry analysis of cell cycle, apoptosis, reactive oxygen species (ROS), and mitochondrial membrane potential (MMP)}

LS180 cells were exposed to $400 \mathrm{nM}$ of GNP-PEG or GNPPEG-R8, again for a $1 \mathrm{~h}$ period. Cells were then washed with PBS and irradiated using $6 \mathrm{MV}$ X-rays at a dose of $6 \mathrm{~Gy}$. For cell-cycle analysis, cells were collected $24 \mathrm{~h}$ postirradiation, fixed in $70 \%$ ethanol at $4^{\circ} \mathrm{C}$ for $24 \mathrm{~h}$, resuspended in PBS, and stained with $50 \mu \mathrm{g} / \mathrm{mL} 7$-aminoactinomycin D (7-AAD) and $10 \mu \mathrm{g} / \mathrm{mL}$ RNase A before incubation at $37^{\circ} \mathrm{C}$ for $30 \mathrm{~min}$. For apoptosis studies, cells were collected $24 \mathrm{~h}$ post-irradiation and incubated for 15 min with $100 \mu \mathrm{L}$ of $1 \times$ buffer solution, $5 \mu \mathrm{L}$ phycoerythrin $(\mathrm{PE})$-Annexin $\mathrm{V}$, and $5 \mu \mathrm{L} 7-\mathrm{AAD}$ at $25^{\circ} \mathrm{C}$. Analysis was performed according to the PE-Annexin-V apoptosis detection kit (BD Biosciences, San Jose, CA, USA) guidelines. For ROS determination, cells were collected $6 \mathrm{~h}$ post-irradiation and treated with dichlorodihydrofluorescein diacetate (DCFH-DA) $(10 \mu \mathrm{mol} / \mathrm{L})$ at $37^{\circ} \mathrm{C}$ in the incubator for $20 \mathrm{~min}$. ROS levels were quantified using an ROS detection kit (Beyotime Biotech, Shang Hai, China). MMP was detected using an MMP assay kit using JC-1 (Beyotime Biotech). Cells were collected $6 \mathrm{~h}$ post-irradiation and stained with $\mathrm{JC}-1$ reagent for $20 \mathrm{~min}$ at $37^{\circ} \mathrm{C}$, before centrifuging $\left(600 \mathrm{~g}\right.$ at $4^{\circ} \mathrm{C}$ for $\left.3 \mathrm{~min}\right)$, washing with JC-1 buffer twice, and resuspending in JC-1 buffer. All samples were subsequently analyzed using a FACSCalibur flow cytometer (BD Biosciences).

\section{Statistical analysis}

A two-tailed Student's $t$-test was used for the statistical analysis, and the value of $p<0.05$ was considered statistically significant. All the experiments were performed as a minimum of three independent replicates, presented as the mean \pm SD. SPSS version 19.0 (IBM Corp., Armonk, NY, USA) was used for all the statistical analyses.

\section{Results \\ Synthesis and characterization of GNP-PEG-R8}

Figure 1A shows representative TEM images of GNP variants. The average GNP core diameter was $6.3 \pm 1.1 \mathrm{~nm}$ (Figure 1B). The TEM image is identical for GNP-PEG-R8 as the peptide cannot be detected by TEM. The hydrodynamic diameter increased to $19.7 \pm 2.8 \mathrm{~nm}$ and $27.8 \pm 1.8 \mathrm{~nm}$ for GNP-PEG and GNP-PEG-R8, respectively, with surface plasmon resonance peaks of $515 \mathrm{~nm}$ for GNP-PEG and $525 \mathrm{~nm}$ for GNP-PEG-R8 (Figures 2 and 3), indicating successful conjugation of the R8 peptide. ${ }^{19,20}$ Furthermore, as shown in Figure S1, the surface charges of GNP-PEG and GNP-PEG-R8 were $-35.0 \pm 5.6 \mathrm{mV}$ and $-29.4 \pm 0.5 \mathrm{mV}$, respectively, again verifying the successful conjugation of the peptide, since the isoelectric point of arginine is 10.76 , so in medium or deionized water, arginine carries a positive charge. To confirm the conjugation of PEG and the R8 peptide on the GNP surface, we performed XPS 

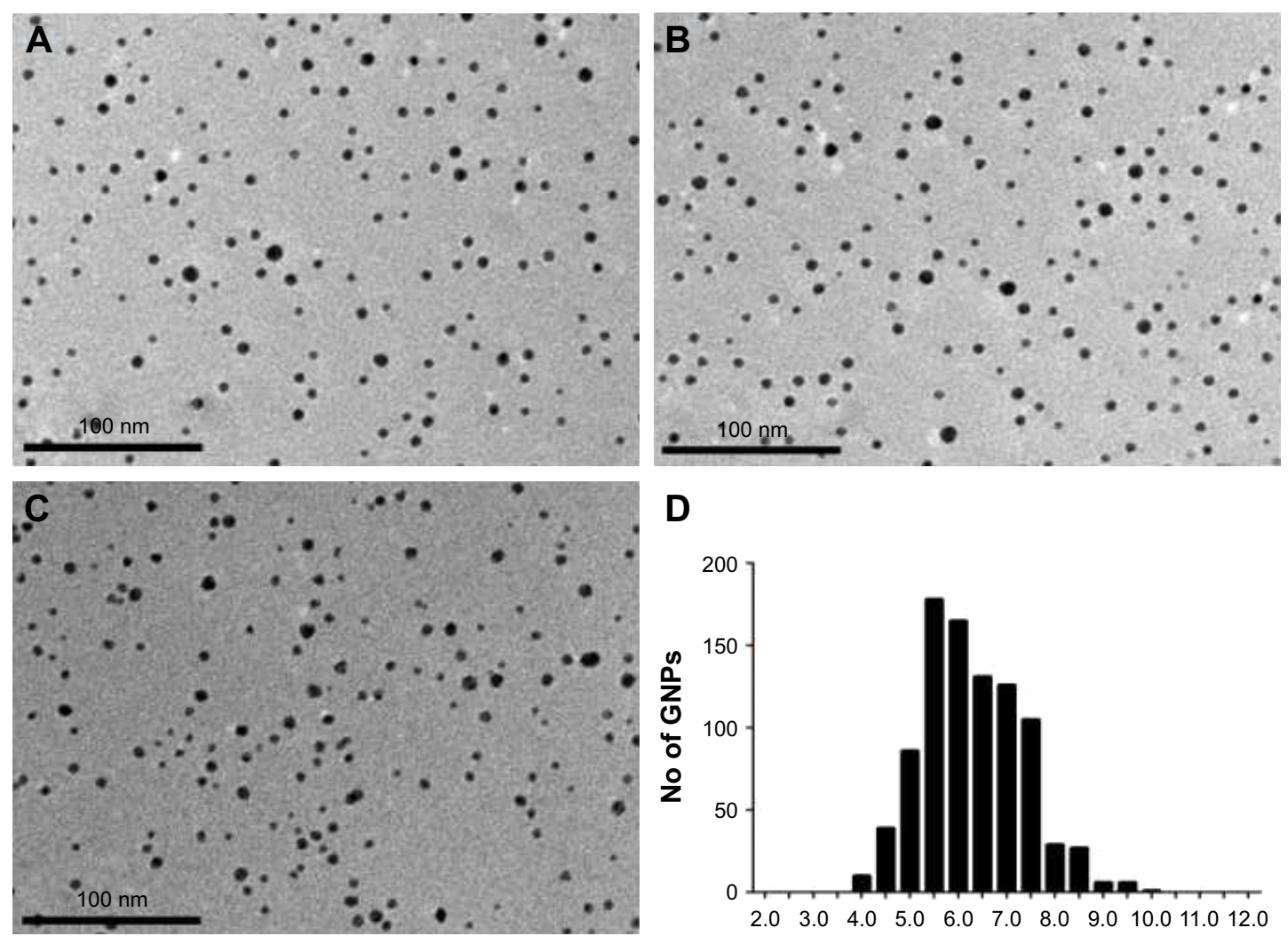

Figure I Characterization of core GNPs. (A-C) TEM image of core GNPs. (D) Size distribution of GNPs determined by ImageJ software. The diameter was $6.3 \pm$ I.I nm (mean \pm SD).

Abbreviations: GNP, gold nanoparticle; TEM, transmission electron microscopy.

analysis. The relative content of nitrogen was 3.7\% for GNPPEG-R8, which was 2.5-fold higher than that for GNP-PEG and the core GNP alone, thus indicating the successful R8 conjugation (Figure 4).

\section{Cytotoxicity assessment}

Direct cytotoxicity conferred by GNP-PEG and GNPPEG-R8 was performed in the absence of radiation, using the
Cell Counting Kit-8 assay. Cytotoxicity was established by drawing comparisons between relative cell viability following exposure to increasing concentrations $(100,200$, $400,800,1,600 \mathrm{nM}$ ) of both GNP variants, compared to the control group (Figure 5). Although the cell viability in the GNP-PEG group was higher than that in the GNP-PEG-R8 group at concentrations of $400 \mathrm{nM}$ and below, there was no significant statistical difference $(p>0.05)$. Furthermore,
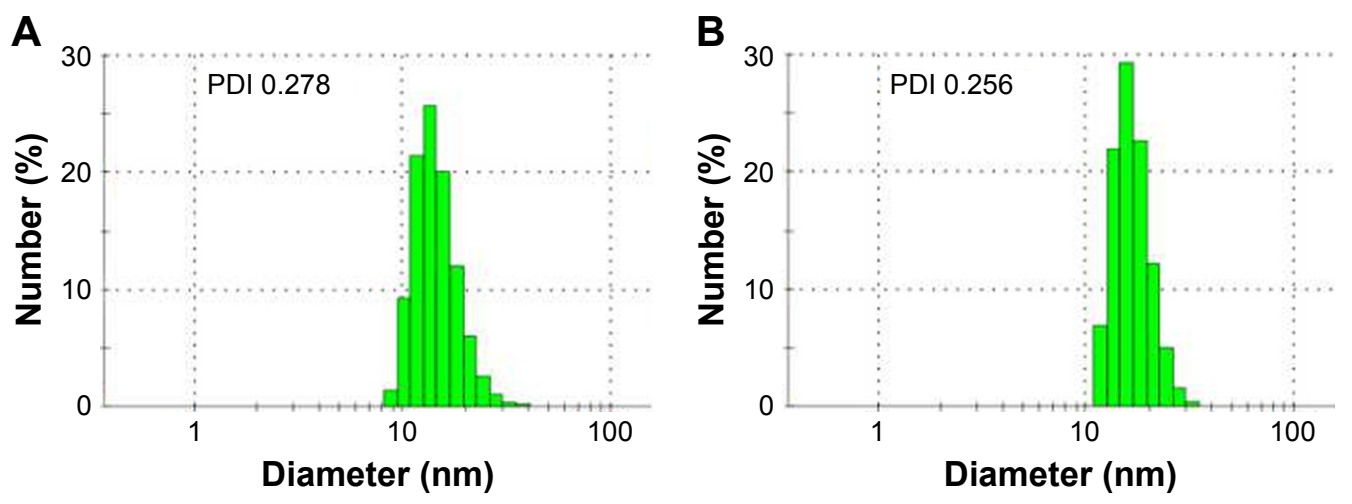

Figure 2 Hydrodynamic diameter distribution of GNP-PEG and GNP-PEG-R8 tested by DLS. (A) Hydrodynamic diameter of GNP-PEG was $19.7 \pm 2.8$ nm (mean \pm SD). (B) Hydrodynamic diameter of GNP-PEG-R8 was $27.8 \pm 1.8 \mathrm{~nm}$ (mean \pm SD).

Abbreviations: GNP, gold nanoparticle; PEG, poly(ethylene glycol); R8, octaarginine; DLS, dynamic light scattering. 


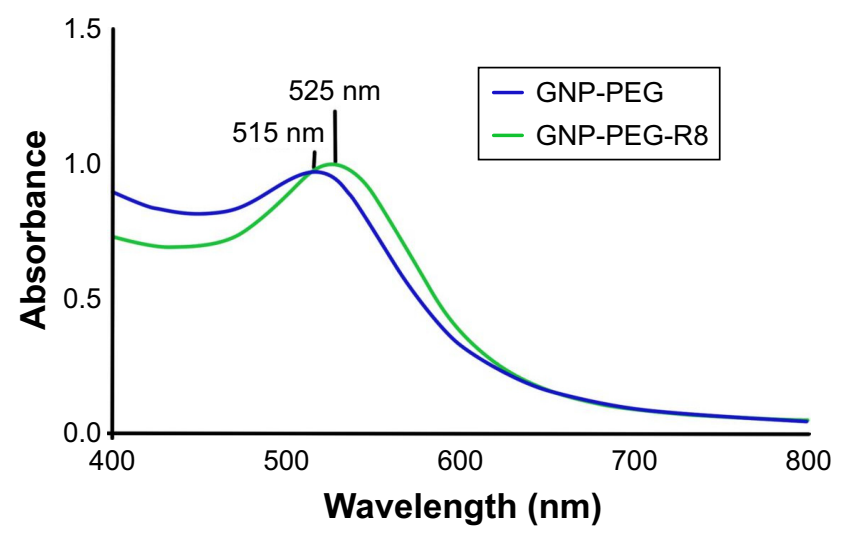

Figure 3 Ultraviolet-visible extinction spectra of GNP-PEG and GNP-PEG-R8 $(400-800 \mathrm{~nm})$. The surface plasmon resonance peaks of GNP-PEG and GNP-PEGR8 were $515 \mathrm{~nm}$ and $525 \mathrm{~nm}$, respectively.

Abbreviations: GNP, gold nanoparticle; PEG, poly(ethylene glycol); R8, octaarginine.

neither GNP variant reduced viability by more than $20 \%$ at concentrations up to $400 \mathrm{nM}$. However, cell viability did appear to reduce in a concentration-dependent manner, dropping to below $80 \%$ survival when treated with $800 \mathrm{nM}$ and 1,600 nM GNP-PEG-R8. As such, a consistent treatment concentration of $400 \mathrm{nM}$ was selected for all subsequent experiments.

\section{Cellular uptake}

Figure 6 represents the cell internalization efficiency of both GNP preparations. Uptake of both GNP-PEG and GNPPEG-R8 occurred in a time-dependent manner, appearing to plateau after a $1 \mathrm{~h}$ incubation. LS180 cells actively internalized GNP-PEG-R8 significantly more efficiently than GNPPEG (2.29 \pm 0.17 vs $0.32 \pm 0.06 \times 10^{5}$ GNP per cell, $\left.p<0.001\right)$, overcoming the negative impact of PEG on cell internalization, an effect coined the "PEG dilemma."21 This response is best illustrated at time-points from $1 \mathrm{~h}$ post-exposure, when an approximate seven-fold increase in intracellular gold was observed for the R8-conjugated GNP in comparison to GNP-PEG. This indicates that increased cellular internalization may be caused by R 8 acting as a transmembrane carrier, thus improving uptake efficiency.

\section{LSI 80 cell radiosensitization conferred by GNP-PEG and GNP-PEG-R8}

The clonogenic assay was used to determine the radiation dose enhancement conferred by the various GNPs. Survival curves for control, GNP-PEG-, and GNP-PEG-R8-treated cells combined with $6 \mathrm{MV}$ X-ray are shown in Figure 7. Table 1 lists the radiobiology parameters of each group, which were derived from fitting the experimental data to the single-hit multi-target model. Unsurprisingly, the surviving fraction decreased with increasing radiation dose, and radiation combined with GNP-PEG-R8 induced a significant reduction in colony formation compared with radiation alone, generating an SER of 1.59. The average lethal dose $D_{0}$ and the quasi-threshold dose $D_{\mathrm{q}}$ in the GNP-PEG-R8 + irradiation (IR) group showed a 1.59-fold and 1.31-fold decrease, respectively, compared with those in the radiation-alone groups. These data have important implications for the protection of normal tissues.

\section{Flow cytometry analysis of cell cycle, apoptosis, ROS levels, and MMP}

The cell-cycle distribution was analyzed to determine whether the radiation enhancement effects were linked to cell-cycle arrest. The data in Figure 8 and Figure S2 demonstrate that compared with radiation alone, there are more cells arrested
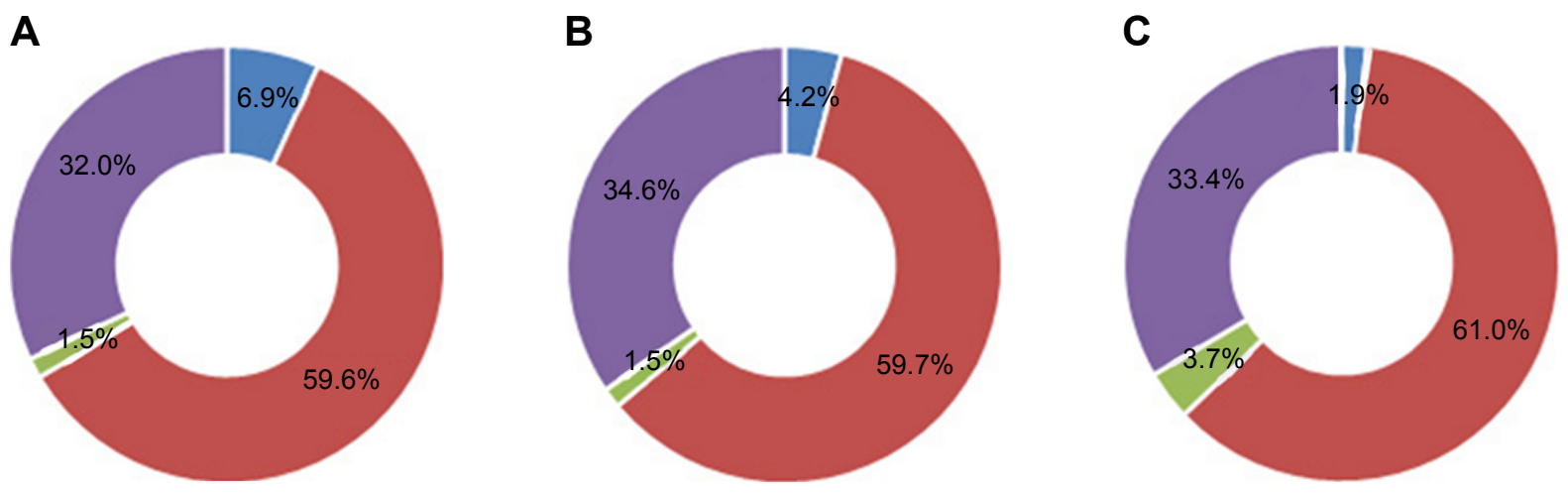

$\square \mathrm{Au} \square \mathrm{C} \square \mathrm{N} \square$ Others

Figure 4 Relative elemental content in GNP-variants analysed by XPS. (A) GNP; (B) GNP-PEG; (C) GNP-PEG-R8. Abbreviations: GNP, gold nanoparticle; XPS, X-ray photoelectron spectroscopy. 


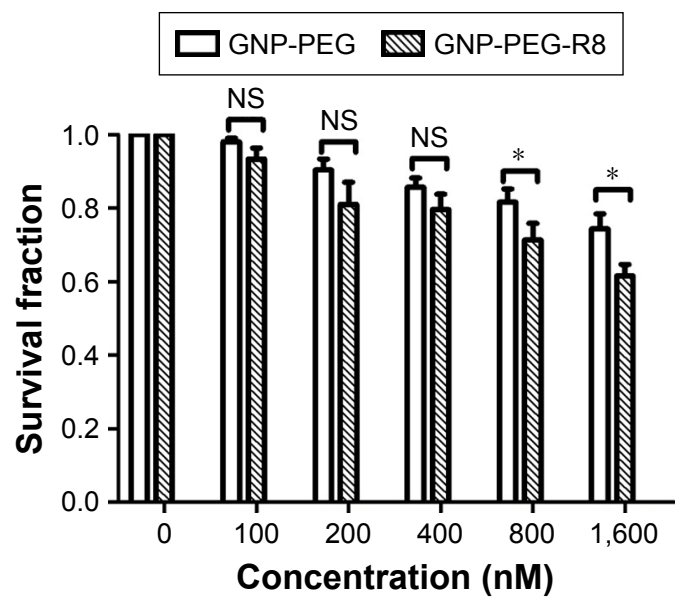

Figure 5 Cell viability of LSI 80 cells after incubation with different concentrations (0, 100, 200, 400, $800 \mathrm{nM})$ of GNP-PEG and GNP-PEG-R8 for $12 \mathrm{~h}$ before examination with the Cell Counting Kit-8 assay. As the concentration increased, the cell viability decreased. Compared with the GNP-PEG group, ${ }^{*} p<0.05$.

Abbreviations: GNP, gold nanoparticle; PEG, poly(ethylene glycol); NS, no significance; R8, octaarginine.

in $\mathrm{G} 2 / \mathrm{M}$ phase following treatment with GNP-PEG-R8 + IR $(32.31 \% \pm 1.84 \%$ vs $41.92 \% \pm 3.28 \%, p<0.05)$. These results suggest that GNP-PEG-R8 enhances the radiosensitivity of LS180 cells through stalling the cell cycle within the G2/M phase.

Figure 9 and Figure $\mathrm{S} 3$ show the percentage of cells undergoing apoptosis following radiation treatment (with or without GNP treatment). There was a significant increase in the percentage of cells undergoing apoptosis in the GNP-PEG-R8 + IR group compared with radiation alone $(57.53 \% \pm 1.68 \%$ vs $10.03 \% \pm 1.43 \%, p<0.001)$. More importantly, compared with GNP-PEG + IR and radiation alone, GNP-PEG-R8 + IR significantly increased the apoptosis rate, corroborating the

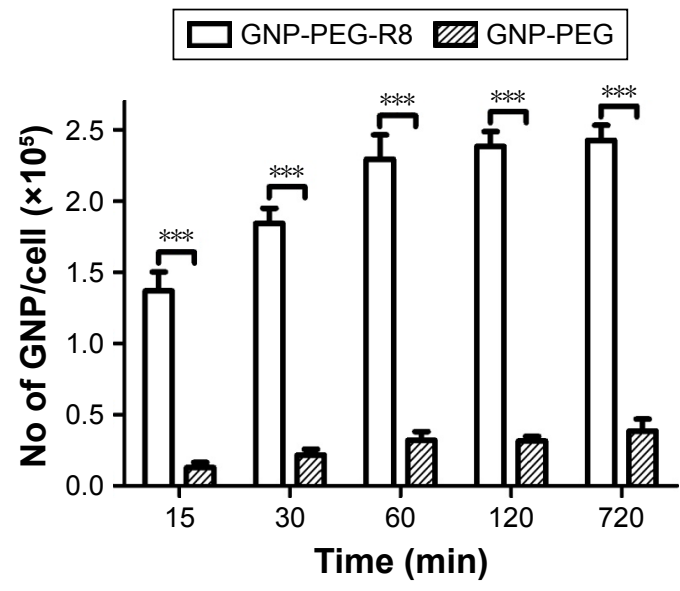

Figure 6 Quantitative analysis of the cellular internalization of GNP-PEG and GNP-PEG-R8 by ICP-MS. Data were quantified and the results are presented as the mean $\pm S D$ ( $n=3$ experiments). Compared with the GNP-PEG group, $* * * p<0.001$. Abbreviations: GNP, gold nanoparticle; PEG, poly(ethylene glycol); R8, octaarginine; ICP-MS, inductively coupled plasma mass spectrometry.

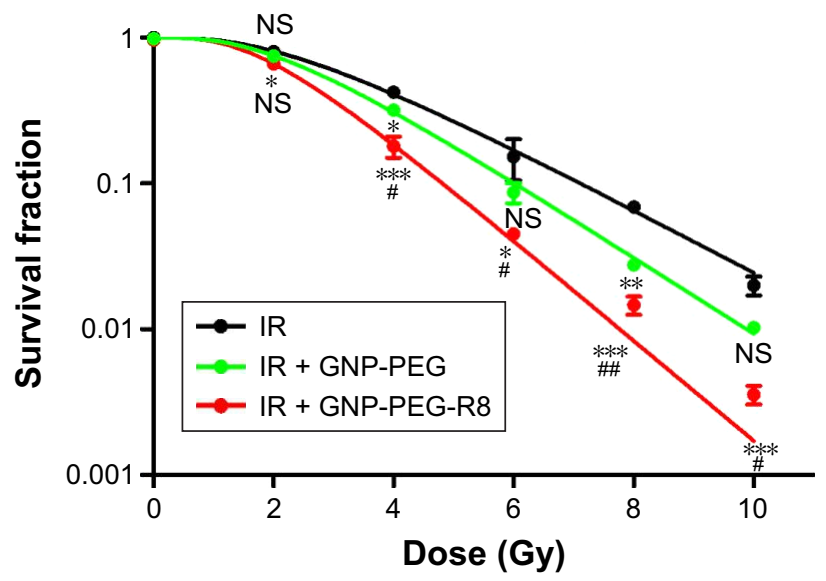

Figure 7 Survival fraction of LSI 80 cells after incubation with GNP-PEG or GNP-PEGR8 (400 nM) for I h before increasing incremental radiation doses $(0,2,4,6,8,10 \mathrm{~Gy})$ of $6 \mathrm{MV} X$-rays ( $\mathrm{n}=3$ experiments). Compared with the IR group, ${ }^{*} p<0.05,{ }^{* *} p<0.01$, and $*_{* * *} p<0.001$. Compared with the IR+ GNP-PEG group, ${ }^{\#} p<0.05$, and ${ }^{\#} p<0.01$.

Abbreviations: GNP, gold nanoparticle; PEG, poly(ethylene glycol); NS, no significance; R8, octaarginine.

cell-cycle data, where complex unresolved DNA lesions at the G2/M checkpoint result in the induction of apoptosis.

Further probing of the underpinning mechanisms driving GNP + IR cell death was determined by measuring intracellular ROS, which, if exceeding the cells' endogenous scavenging capacity, can result in oxidative damage to intracellular biomolecules, promoting damage and death. ROS levels were measured using the DCFH-DA fluorescence method. Figure 10 illustrates a highly significant increase in ROS following GNP-PEG-R8 + IR treatment compared with radiation alone $(p<0.001)$. Importantly, this was about three- and four-fold greater than that measured in the GNPPEG + IR and radiation-alone groups. This result suggests that elevated ROS following GNP-PEG-R8 is the likely mediator of both treatment-induced cell-cycle stalling and apoptosis leading to enhanced radiosensitivity.

Finally, as the release of cytochrome $c$ following mitochondrial membrane depolarization is central to the induction of intrinsic apoptosis, we measured the extent to which the GNP variants could trigger depolarization. As shown in Figure 11, in unirradiated groups, GNP-PEG and

Table I Radiobiology parameters of each group, calculated using the single-hit multi-target model

\begin{tabular}{llllllll}
\hline & $D_{0}(G y)$ & $D_{q}(G y)$ & $S F_{2}$ & & & \\
\cline { 5 - 7 } & & & & & $D_{0}(G y)$ & $D_{q}(G y)$ & $S F_{2}$ \\
\hline IR & 2.01 & 2.56 & 0.80 & & & \\
IR + GNP-PEG & 1.65 & 2.26 & 0.74 & 1.21 & 1.13 & 1.07 \\
IR + GNP-PEG-R8 & 1.27 & 1.96 & 0.59 & 1.59 & 1.31 & 1.36 \\
\hline
\end{tabular}

Abbreviations: IR, irradiation; GNP, gold nanoparticle; PEG, poly(ethylene glycol); $R 8$, octaarginine; $D_{0}$, average lethal dose; $D_{q}$, quasi-threshold dose; $S F_{2}$, survival fraction at radiation dose of $2 \mathrm{~Gy}$. 


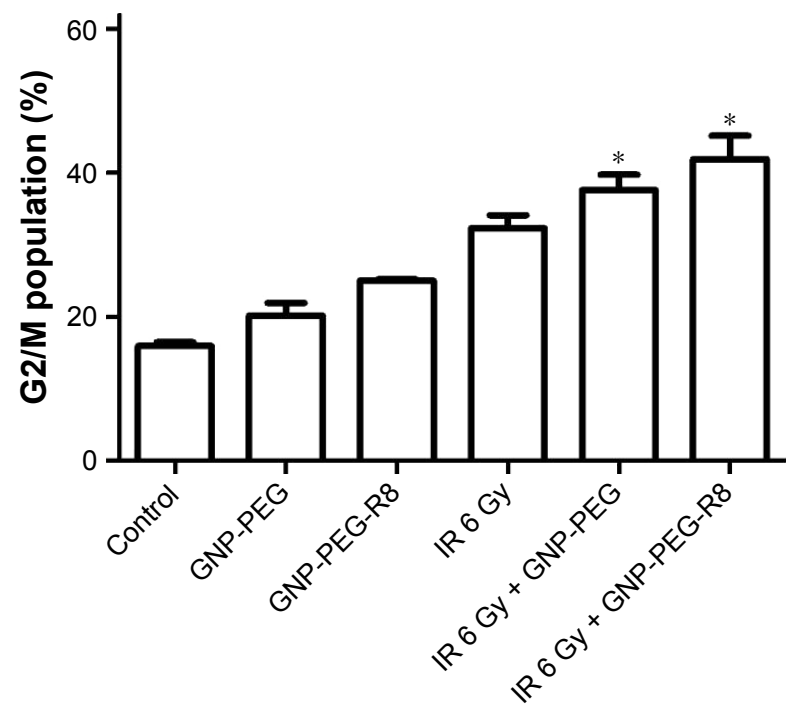

Figure 8 Flow cytometry analysis of G2/M cell-cycle arrest in LSI80 cells. Data were quantified and the results are presented as the mean $\pm S D$. Compared with the IR 6 Gy group, * $p<0.05$.

Abbreviations: GNP, gold nanoparticle; PEG, poly(ethylene glycol); R8, octaarginine.

GNP-PEG-R8 slightly suppressed mitochondrial membrane polarization relative to the untreated control $(14.97 \% \pm 0.25 \%$, $12.60 \% \pm 0.75 \%$, and $6.80 \% \pm 0.36 \%$, respectively). The MMP decreased for cells treated with radiation alone $(28.17 \% \pm 2.50 \%)$. However, highly significant increases in membrane depolarization were observed, for the GNPPEG + IR group $(43.73 \% \pm 4.63 \%)$ and particularly for the GNP-PEG-R8 + IR group $(67.87 \% \pm 7.24 \%, p<0.001)$ compared with radiation alone.

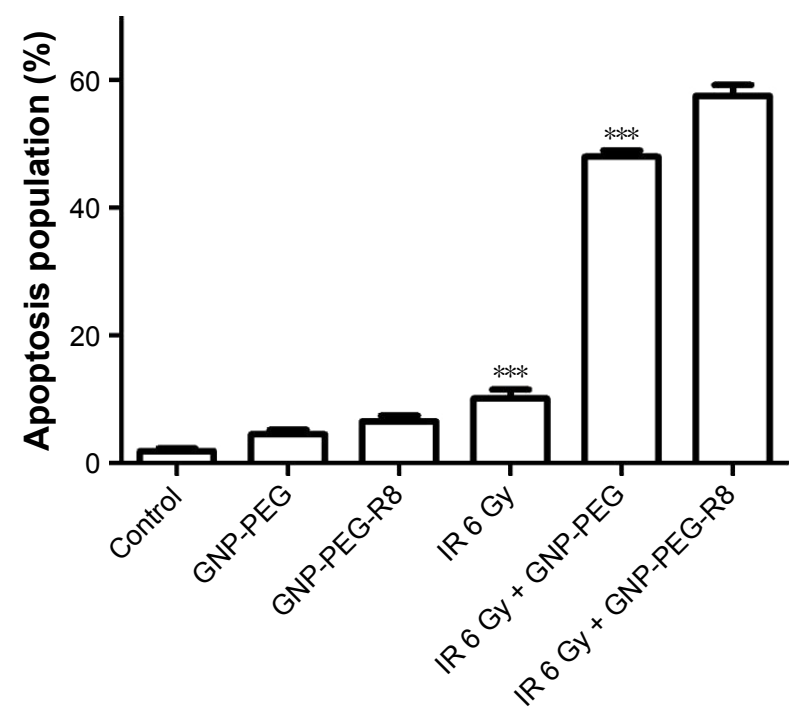

Figure 9 Flow cytometry analysis of apoptosis in LSI80 cells. Data were quantified and the results are presented as the mean \pm SD. Compared with the IR 6 Gy + GNPPEG-R8 group, $* * * p<0.001$.

Abbreviations: IR, irradiation; GNP, gold nanoparticle; PEG, poly(ethylene glycol); R8, octaarginine.

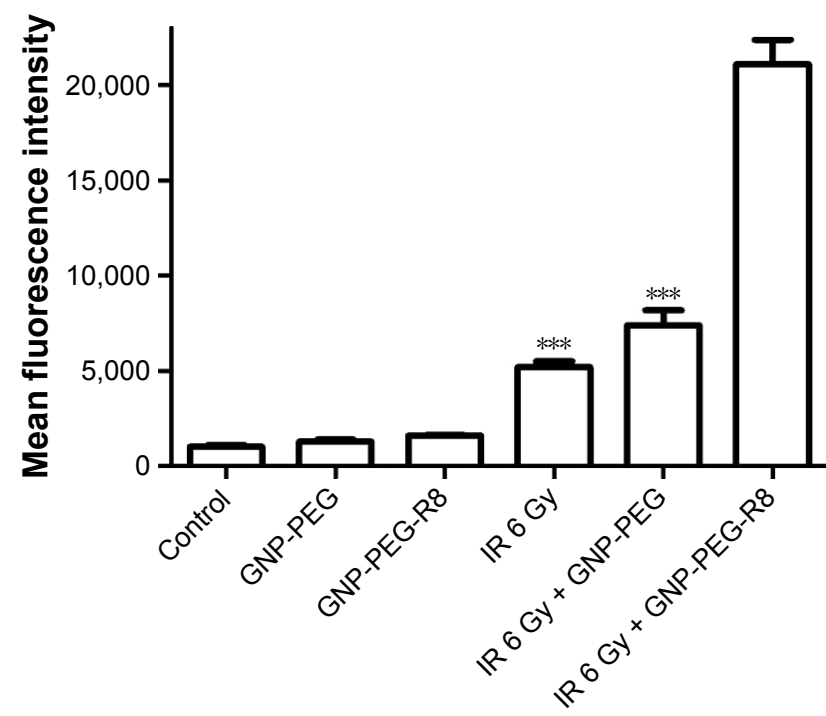

Figure 10 ROS levels in LSI80 cells treated with GNP-PEG or GNP-PEG-R8 with or without $6 \mathrm{MV}$ X-rays (6 Gy) as determined by flow cytometry. Data were quantified and the results are presented as the mean $\pm S D$. Compared with the IR 6 Gy + GNP-PEG-R8 group, ${ }^{* * *} p \leq 0.001$.

Abbreviations: ROS, reactive oxygen species; IR, irradiation; GNP, gold nanoparticle; PEG, poly(ethylene glycol); R8, octaarginine.

\section{Discussion}

The development of advanced radiotherapy techniques such as intensity-modulated radiotherapy, image-guided radiotherapy, and tomotherapy has revolutionized clinical radiotherapy, delivering personalized treatments to maximize patient response. However, dose-limiting toxicities remain one of the key obstacles impeding the success of radiotherapy.

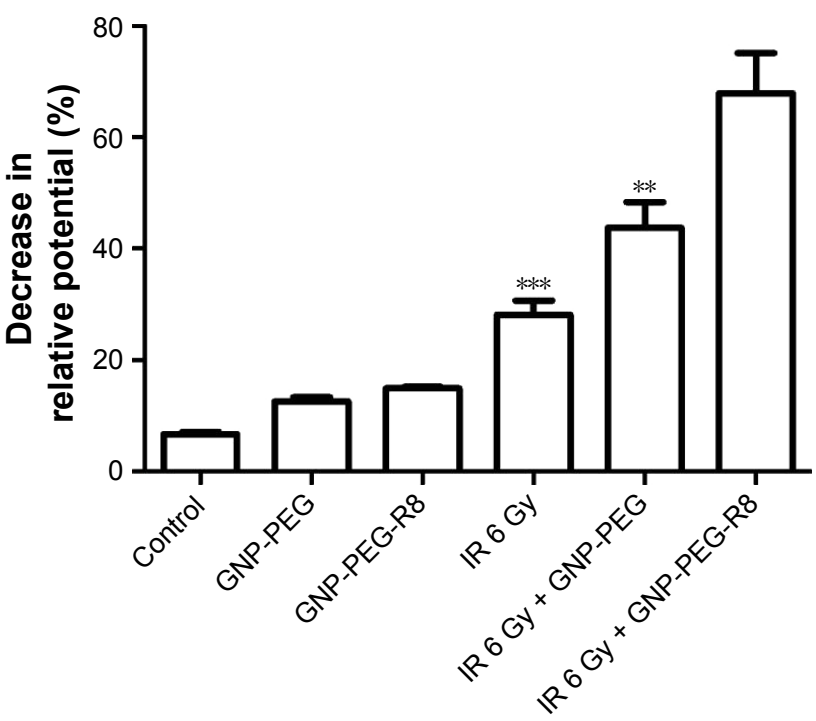

Figure II Mitochondrial membrane potential analysis in LSI80 cells. Data were quantified and the results are presented as the mean $\pm S D$. Compared with the IR 6 Gy + GNP-PEG-R8 group, ${ }^{* *} p<0.01$, *** $p<0.001$.

Abbreviations: ROS, reactive oxygen species; IR, irradiation; GNP, gold nanoparticle; PEG, poly(ethylene glycol); R8, octaarginine. 
Since Hainfeld et al presented the frst study using GNP as a radiosensitizer, about fourteen years ago, various nanoparticles have been developed, which have been reported to enhance the efficacy of radiotherapy within multiple tumor types. ${ }^{12,13,22-24}$ The current study presents an alternative strategy for increasing the radiosensitization potential of GNP through the conjugation of an arginine-rich CPP.

Despite the significant body of evidence supporting GNP as an effective radiosensitizer, high treatment concentrations and long incubation times limit clinical translation. In this study, GNP-PEG-R8 were demonstrated to penetrate the cell membrane with high efficiency. Over $90 \%$ of total uptake occurred within the first hour, and, importantly, internal GNP concentrations were sustained at this level for at least $12 \mathrm{~h}$. This may be due to the positive charge of the peptide, which promotes electrostatic interactions with the negatively charged cell membrane. Although numerous studies exist relating to the uptake mechanism of CPPs across the plasma membrane, the precise mechanism is yet to be fully understood. ${ }^{25}$ However, for arginine-rich CPPs, evidence shows that the dominant uptake mechanism is direct membrane translocation as opposed to receptor-mediated endocytosis. ${ }^{26}$ If this is the case for the GNP-PEG-R8 nanoparticle, it may help to explain the high efficiency of GNP-PEG-R8 internalization, since endocytosis is a complex multistep process. Furthermore, a direct translocation mechanism is not thought to induce significant membrane damage, thereby preserving cell viability, as observed, at the relevant treatment concentration. ${ }^{15}$

Clonogenic survival data demonstrated that GNPPEG-R8 exerted a significant reduction in colony-forming potential compared to GNP-PEG, demonstrating that R8 conjugation enhanced the sensitivity of LS180 cells to megavoltage radiation. Furthermore, the quasi-threshold dose $D_{\mathrm{q}}$ represents the ability for sublethal damage repair, which is proportional to $D_{\mathrm{q}}$. The decrease in $D_{\mathrm{q}}$ in cancer is highly important with respect to improved local control. Specifically, GNP-PEG-R8 shifted the average lethal dose $D_{0}$ decrease from $2.01 \mathrm{~Gy}$ in radiation only cells to $1.27 \mathrm{~Gy}$ in GNP-PEG-R8 + IR, offering meaningful protection for normal tissues against radiation induced damage. The underlying mechanisms by which GNP-PEG-R8 delivered impressive radiation dose enhancement were correlated with several biological factors.

Several groups have established that the sensitivity of cells to radiation is closely correlated with the cell cycle and the induction of apoptosis. ${ }^{27,28}$ Therefore, we examined these parameters following treatment with GNP-PEG-R8. Cellcycle analysis showed that the combination of radiation and
GNP-PEG-R8 had an additive effect yielding an increased proportion of cells in $\mathrm{G} 2 / \mathrm{M}$ phase, a result consistent with previous studies. ${ }^{28,29}$ Tumor cells in G2/M are more sensitive to radiation, while cells in G1/S are less sensitive, resisting the effects of radiation. Therefore, GNP-PEG-R8 combined with radiation has the potential to induce more damage to the target cells.

Radiation interacts with intracellular molecules, predominantly water, to generate free radicals. In general, excessive ROS disrupts cellular function, causing lipid, protein, and DNA oxidation, and triggering cell death through various downstream signaling pathways. ${ }^{30,31}$ These factors act as a potential trigger for radiation-induced apoptosis. ${ }^{30}$ Our results show that intracellular ROS levels in the GNP-PEG-R8 + IR group were markedly higher than those observed in all other treatment groups, yielding a higher level of oxidative stress, manifested as enhanced apoptosis compared to radiation alone. This result clearly indicates that increased intracellular ROS is a key mechanism mediating GNP-PEG-R8 radiosensitization.

To further establish the underlying mechanisms of GNP-mediated radiosensitization, we tested mitochondrial membrane depolarization by flow cytometry. Mitochondria function within cells by contributing to bioenergetics, metabolism, and biosynthesis. ${ }^{32-34}$ Loss of MMP is associated with many pathophysiological changes. ${ }^{35,36}$ In this study, we demonstrated that GNP-PEG-R8 combined with radiation had a more significant impact on mitochondrial membrane depolarization than any other group, indicating that mitochondria are particularly susceptible to oxidative damage. Mitochondrial membrane depolarization can be caused by the presence of ROS, high intracellular calcium concentrations, or stress of the endoplasmic reticulum. ${ }^{37} \mathrm{We}$ report increased ROS after irradiation with GNPs, corroborating our findings that elevated ROS results in mitochondrial depolarization contributing towards GNP-related radiosensitization. Furthermore, mitochondrial membrane depolarization leads to a sudden increase in the permeability of the inner mitochondrial membrane, resulting in the release of cytochrome $c$, caspase activation, and the induction of apoptosis. ${ }^{38}$ In summary, ROS may be the initiator and mitochondrial damage may represent a key extranuclear target, regulating GNPs plus IR-mediated cell death. In consideration of the effects of mitochondrial dysfunction, future studies could focus on mitochondrial-specific delivery of GNPs to further improve radiation enhancement.

Despite the potential of GNPs to confer enhanced radiosensitivity, there remain several challenges towards clinical translation. Future studies will be conducted with the goal 
of reducing long-term side effects in vivo, achieving higher stability, specific intracellular organelle targeting, or tumor microenvironment targeting by modifying GNPs with additional moieties such as nuclear or mitochondrial targeting sequence. Furthermore, in view of the impressive efficacy of GNP-PEG-R8 internalization, variants of this preparation could be used as a drug-delivery platform. Conjugation of cytotoxic chemotherapy drugs could help to improve accurate delivery and release of drug cargos, leading to a widening of their therapeutic window along with a corresponding reduction in off-target side effects.

\section{Conclusion}

In this study, we demonstrated that R8-modified GNPs could be efficiently internalized by LS180 cells, within $1 \mathrm{~h}$ of treatment, while conveying little cytotoxicity. Importantly, incorporation of the R8 CPP increased GNP uptake by almost seven-fold over GNP-PEG, leading to significant radiosensitization effects in combination with megavoltage radiation treatment in vitro. GNP-PEG-R8 may enhance radiosensitivity by causing cell-cycle disruption and inducing apoptosis, with elevated ROS identified as the likely initiator.

\section{Acknowledgments}

The human colorectal cancer cell line LS180 was kindly gifted by the Institute of Zoology, Chinese Academy of Sciences. This work was supported by the National Natural Science Foundation of China (no 81372420).

\section{Disclosure}

The authors report no conflicts of interest in this work.

\section{References}

1. Boisselier E, Astruc D. Gold nanoparticles in nanomedicine: preparations, imaging, diagnostics, therapies and toxicity. Chem Soc Rev. 2009; 38(6):1759-1782.

2. Ngwa W, Kumar R, Sridhar S, et al. Targeted radiotherapy with nanoparticles: current status and future perspectives. Nanomedicine (Lond). 2014;9(7):1063-1082.

3. Cuenca AG, Jiang H, Hochwald SN, Delano M, Cance WG, Grobmyer SR. Emerging implications of nanotechnology on cancer diagnostics and therapeutics. Cancer. 2006;107(3):459-466.

4. Liong M, Lu J, Kovochich M, et al. Multifunctional inorganic nanoparticles for imaging, targeting, and drug delivery. ACS Nano. 2008; 2(5):889-896.

5. Dou Y, Guo Y, Li X, et al. Size-Tuning Ionization To Optimize Gold Nanoparticles for Simultaneous Enhanced CT Imaging and Radiotherapy. ACS Nano. 2016;10(2):2536-2548.

6. Zhang X, Wu D, Shen X, et al. Size-dependent radiosensitization of PEGcoated gold nanoparticles for cancer radiation therapy. Biomaterials. 2012;33(27):6408-6419.

7. Mármol I, Sánchez-de-Diego C, Pradilla Dieste A, Cerrada E, Rodriguez Yoldi MJ. Colorectal Carcinoma: A General Overview and Future Perspectives in Colorectal Cancer. Int J Mol Sci. 2017;18(1):197.
8. National Comprehensive Cancer Network. The NCCN colon cancer clinical practice guidelines in oncology (version 1.2017). Available from: https://www.nccn.org/professionals/physician_gls/default. aspx\#colon. Accessed November 10, 2017.

9. Seo YS, Kim MS, Yoo HJ, Jang WI. Stereotactic body radiotherapy for oligo-recurrence within the nodal area from colorectal cancer. World $J$ Gastroenterol. 2014;20(8):2005-2013.

10. Shi M, Paquette B, Thippayamontri T, Gendron L, Guérin B, Sanche L. Increased radiosensitivity of colorectal tumors with intra-tumoral injection of low dose of gold nanoparticles. Int J Nanomedicine. 2016;11: 5323-5333.

11. Jain S, Coulter JA, Hounsell AR, et al. Cell-specific radiosensitization by gold nanoparticles at megavoltage radiation energies. Int J Radiat Oncol Biol Phys. 2011;79(2):531-539.

12. Hainfeld JF, Dilmanian FA, Slatkin DN, Smilowitz HM. Radiotherapy enhancement with gold nanoparticles. J Pharm Pharmacol. 2008; 60(8):977-985.

13. Zhao N, Yang Z, Li B, et al. RGD-conjugated mesoporous silicaencapsulated gold nanorods enhance the sensitization of triple-negative breast cancer to megavoltage radiation therapy. Int $J$ Nanomedicine. 2016;11:5595-5610.

14. Farkhani SM, Valizadeh A, Karami H, Mohammadi S, Sohrabi N, Badrzadeh F. Cell penetrating peptides: efficient vectors for delivery of nanoparticles, nanocarriers, therapeutic and diagnostic molecules. Peptides. 2014;57:78-94.

15. Wang F, Wang Y, Zhang X, Zhang W, Guo S, Jin F. Recent progress of cell-penetrating peptides as new carriers for intracellular cargo delivery. J Control Release. 2014;174:126-136.

16. Ramsey JD, Flynn NH. Cell-penetrating peptides transport therapeutics into cells. Pharmacol Ther. 2015;154:78-86.

17. Tünnemann G, Ter-Avetisyan G, Martin RM, Stöckl M, Herrmann A, Cardoso MC. Live-cell analysis of cell penetration ability and toxicity of oligo-arginines. J Pept Sci. 2008;14(4):469-476.

18. Frens G. Controlled nucleation for the regulation of the particle size in monodisperse gold suspensions. Nat Phys Sci. 1973;241(105):20-22.

19. Wang Y, Ni Y. Combination of UV-vis spectroscopy and chemometrics to understand protein-nanomaterial conjugate: a case study on human serum albumin and gold nanoparticles. Talanta. 2014;119: 320-330.

20. Piliarik M, Homola J. Surface plasmon resonance (SPR) sensors: approaching their limits? Opt Express. 2009;17(19):16505-16517.

21. Liu T, Thierry B. A solution to the PEG dilemma: efficient bioconjugation of large gold nanoparticles for biodiagnostic applications using mixed layers. Langmuir. 2012;28(44):15634-15642.

22. Her S, Cui L, Bristow RG, Allen C. Dual action enhancement of gold nanoparticle radiosensitization by pentamidine in triple negative breast cancer. Radiat Res. 2016;185(5):549-562.

23. Wolfe T, Chatterjee D, Lee J, et al. Targeted gold nanoparticles enhance sensitization of prostate tumors to megavoltage radiation therapy in vivo. Nanomedicine. 2015;11(5):1277-1283.

24. Hainfeld JF, Slatkin DN, Smilowitz HM. The use of gold nanoparticles to enhance radiotherapy in mice. Phys Med Biol. 2004;49(18): N309-N315.

25. Fonseca SB, Pereira MP, Kelley SO. Recent advances in the use of cell-penetrating peptides for medical and biological applications. $A d v$ Drug Deliv Rev. 2009;61(11):953-964.

26. Liu BR, Huang Y, Winiarz JG, Chiang H, Lee H. Intracellular delivery of quantum dots mediated by a histidine- and arginine-rich HR9 cell-penetrating peptide through the direct membrane translocation mechanism. Biomaterials. 2011;32(13):3520-3537.

27. Mahmoudi M, Azadmanesh K, Shokrgozar MA, Journeay WS, Laurent S. Effect of nanoparticles on the cell life cycle. Chem Rev. 2011; 111(5):3407-3432.

28. Roa W, Zhang X, Guo L, et al. Gold nanoparticle sensitize radiotherapy of prostate cancer cells by regulation of the cell cycle. Nanotechnology. 2009;20(37):375101. 
29. Wang C, Jiang Y, Li X, Hu L. Thioglucose-bound gold nanoparticles increase the radiosensitivity of a triple-negative breast cancer cell line (MDA-MB-231). Breast Cancer. 2015;22(4):413-420.

30. Zhang J, Wang X, Vikash V, et al. ROS and ROS-mediated cellular signaling. Oxid Med Cell Longev. 2016;2016:4350965.

31. Sabharwal SS, Schumacker PT. Mitochondrial ROS in cancer: initiators, amplifiers or an Achilles' heel? Nat Rev Cancer. 2014;14(11): 709-721.

32. Rama Rao KV, Norenberg MD. Brain energy metabolism and mitochondrial dysfunction in acute and chronic hepatic encephalopathy. Neurochem Int. 2012;60(7):697-706.

33. Melser S, Lavie J, Bénard G. Mitochondrial degradation and energy metabolism. Biochim Biophys Acta. 2015;1853(10):2812-2821.

34. Kehrein K, Bonnefoy N, Ott M. Mitochondrial protein synthesis: efficiency and accuracy. Antioxid Redox Signal. 2013;19(16):1928-1939.
35. Jin SM, Lazarou M, Wang C, Kane LA, Narendra DP, Youle RJ. Mitochondrial membrane potential regulates PINK1 import and proteolytic destabilization by PARL. J Cell Biol. 2010;191(5):933-942.

36. Kadenbach B, Ramzan R, Moosdorf R, Vogt S. The role of mitochondrial membrane potential in ischemic heart failure. Mitochondrion. 2011;11(5):700-706.

37. Deniaud A, Sharaf el dein O, Maillier E, et al. Endoplasmic reticulum stress induces calcium-dependent permeability transition, mitochondrial outer membrane permeabilization and apoptosis. Oncogene. 2008;27(3):285-299.

38. GerasimenkoJV, GerasimenkoOV,Palejwala A, Tepikin AV,PetersenOH, Watson A. Menadione-induced apoptosis: roles of cytosolic $\mathrm{Ca}(2+)$ elevations and the mitochondrial permeability transition pore. $J$ Cell Sci. 2002;115(3):485-497. 


\section{Supplementary materials}
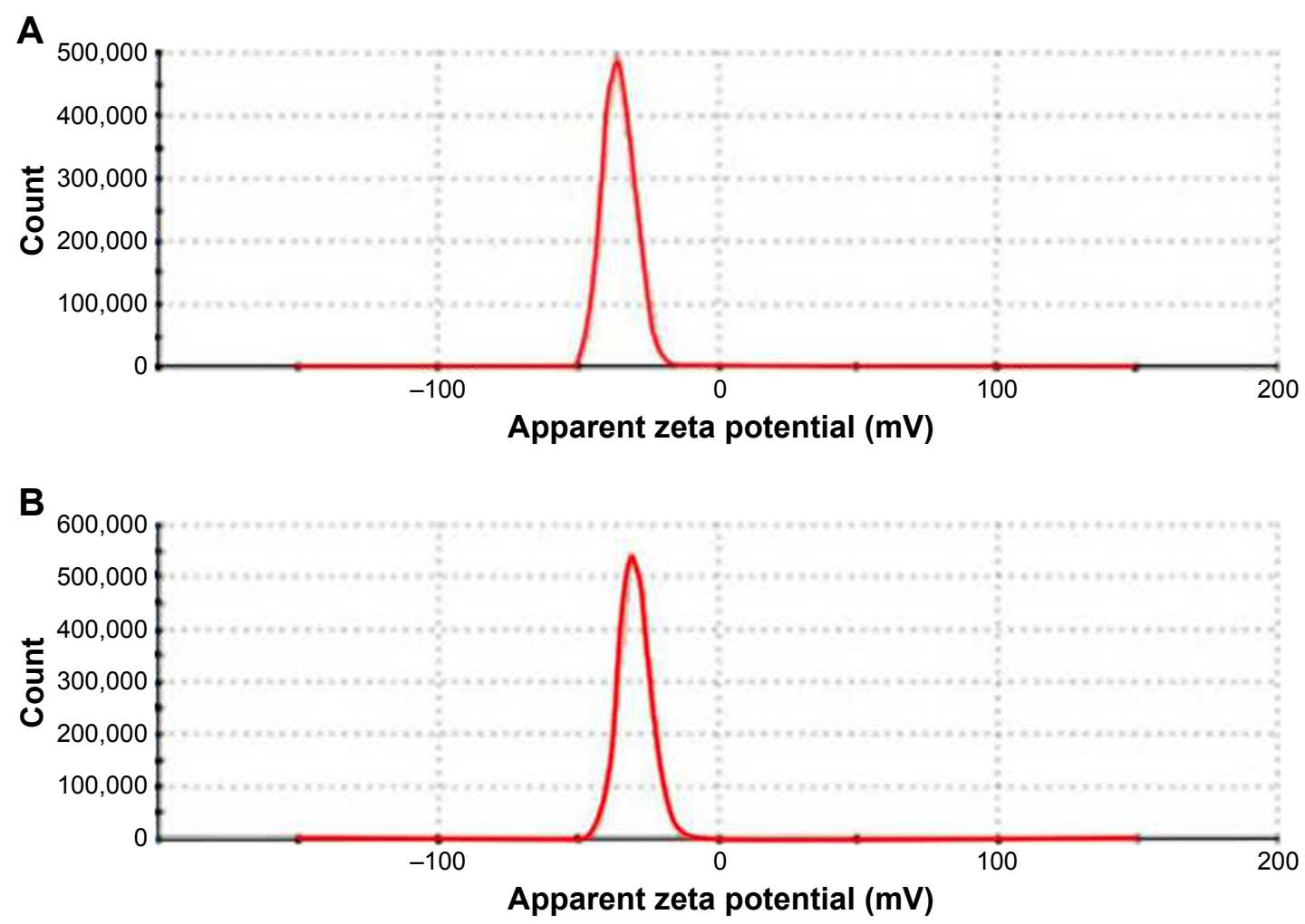

Figure SI Zeta potential of (A) GNP-PEG and (B) GNP-PEG-R8. The zeta potentials of GNP-PEG and GNP-PEG-R8 were $-35.0 \pm 5.6 \mathrm{mV}$ and $-29.4 \pm 0.5 \mathrm{mV}$, respectively. Abbreviations: GNP, gold nanoparticle; PEG, poly(ethylene glycol); R8, octaarginine.
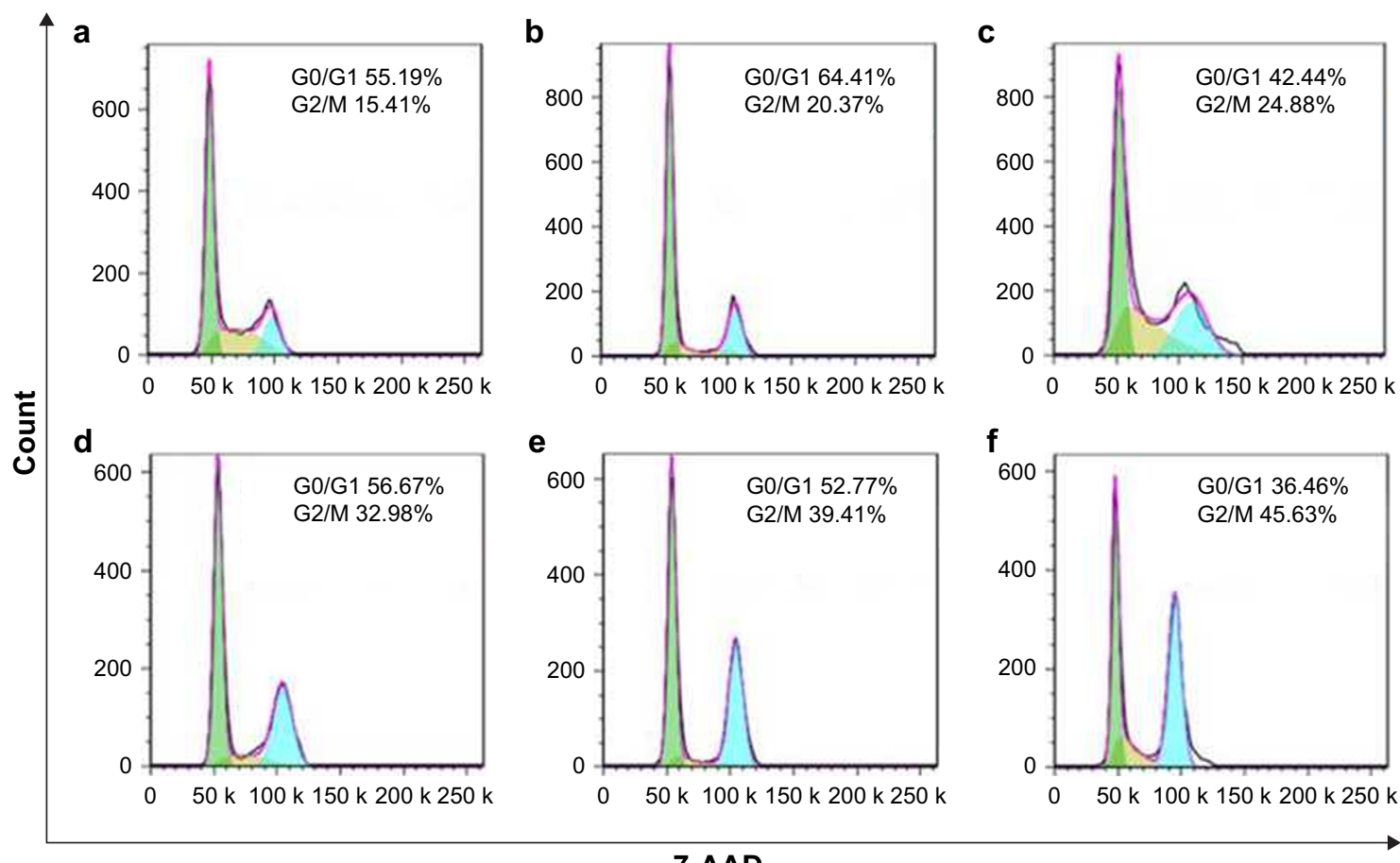

Figure S2 Flow cytometry analysis of cell-cycle arrest in LSI 80 cells. (a) Control group; (b) GNP-PEG group; (c) GNP-PEG-R8 group; (d) IR group; (e) IR 6 Gy + GNP-PEG group; (f) IR 6 Gy + GNP-PEG-R8 group.

Abbreviations: GNP, gold nanoparticle; PEG, poly(ethylene glycol); R8, octaarginine; IR, irradiation. 

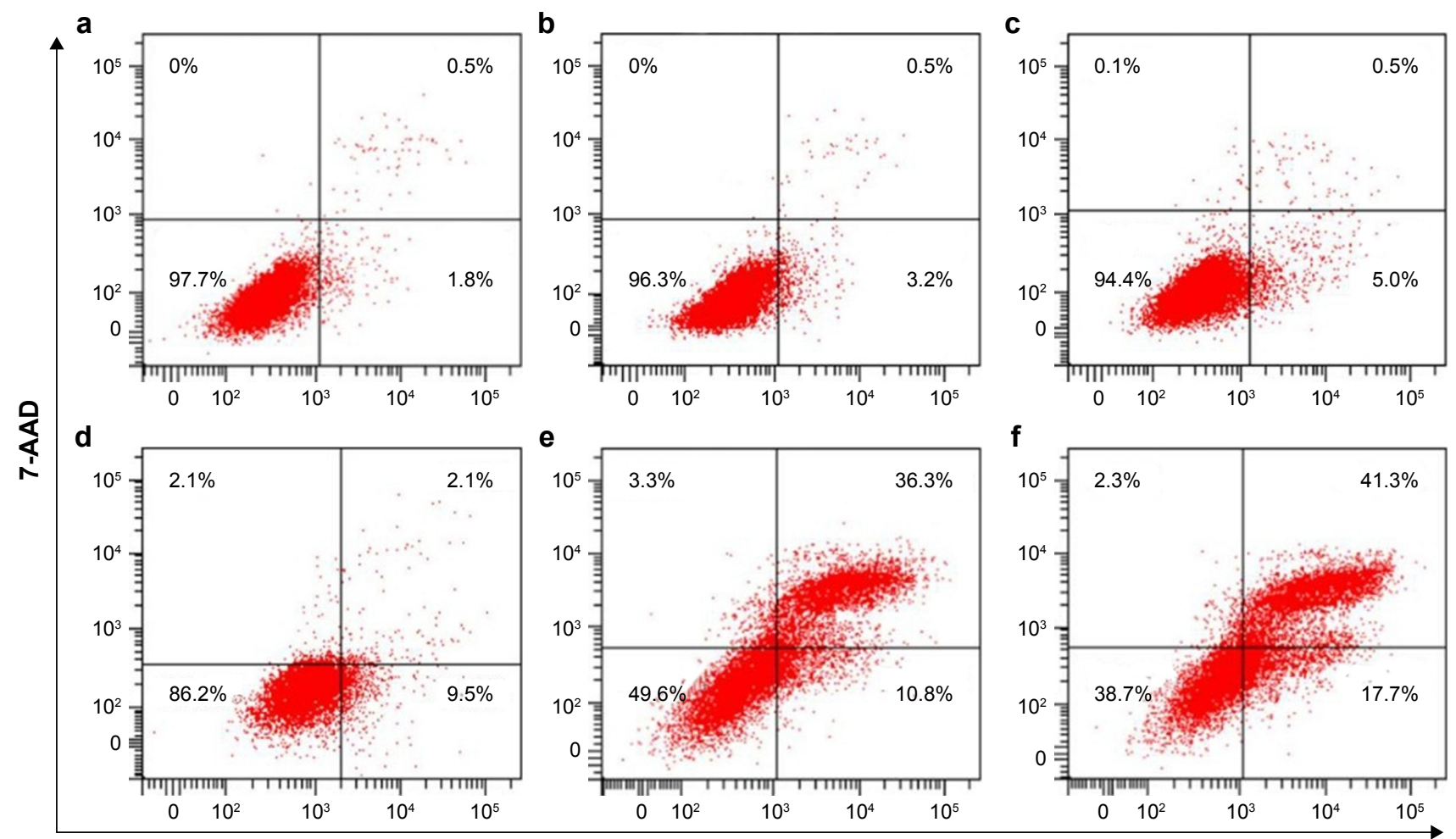

Annexin-V PE

Figure S3 Flow cytometry analysis of apoptosis in LSI 80 cells. (a) Control group; (b) GNP-PEG group; (c) GNP-PEG-R8 group; (d) IR group; (e) IR 6 Gy + GNP-PEG group; (f) IR 6 Gy + GNP-PEG-R8 group.

Abbreviations: GNP, gold nanoparticle; PEG, poly(ethylene glycol); R8, octaarginine; IR, irradiation; 7-AAD, aminoactinomycin D; PE, phycoerythrin.

\section{Publish your work in this journal}

The International Journal of Nanomedicine is an international, peerreviewed journal focusing on the application of nanotechnology in diagnostics, therapeutics, and drug delivery systems throughout the biomedical field. This journal is indexed on PubMed Central, MedLine, CAS, SciSearch $®$, Current Contents ${ }^{\circledR} /$ Clinical Medicine,
Journal Citation Reports/Science Edition, EMBase, Scopus and the Elsevier Bibliographic databases. The manuscript management system is completely online and includes a very quick and fair peer-review system, which is all easy to use. Visit http://www.dovepress.com/ testimonials.php to read real quotes from published authors. 